\title{
Prevalence and risk factors for chest-related symptoms of acute respiratory tract infections among under five children: Case of Ethiopia
}

\author{
Berhanu Teshome Woldeamanuel* and Haftu Legesse Gebreyesus \\ Department of Statistics, Mekelle University, P.O. Box231, Mekelle, Tigray, Ethiopia
}

\begin{abstract}
Background: Acute respiratory infection is a major public health problem to morbidity and mortality among under five children. Globally it is estimated that about 7.6 million children were died before celebrating their fifth year of birth day attributed to respiratory tract infections, where 3.6 million deaths were in Africa and about 2.1 million deaths were in Southeast Asia. In Ethiopia acute respiratory infections are major problem accounting for about $10 \%$ of under-five deaths each year.

Objective: This study was an attempt to study the prevalence and risk factors of acute respiratory infections in Ethiopia using data collected in Ethiopian demographic and health survey 2016 .

Methods: Descriptive statistics and multivariate binary logistic regression were used considering child characteristics, maternal socioeconomic, demographic characteristics, environmental and health factors variables as explanatory variables and symptoms of acute respiratory infection as the response variable.

Results: Prevalence of acute respiratory infection among under-five children was $10.4 \%$. Results of logistic regression analysis shows that place of residence, sex of household head, education level of mother, partners education, baby postnatal checkup, source of drinking water, age of a child in months, and working status of mother are found to be the significant risk factors for occurrence of ARI symptoms among under five children at 5\% level of significance. While the variables sex of a child, availability of toilet facility, wealth index, age of mother at first birth, family size, and birth order of a child are not statistically significant at $5 \%$ level of significance.
\end{abstract}

Conclusion: Children from rural areas and illiterate mothers are more at risk for acute respiratory infection. Thus, interventions should target mothers with less education and those residing in rural areas for better care for their children.

Abbreviations: ARI: Acute Respiratory Infection; CI: Confidence Interval; EDHS: Ethiopian Demographic and Health Survey; GBD: Global Burden of Diseases; HIV/AIDS: Human Immune Virus/ Acquired Immune Deficiency Syndrome; IRLS: Iteratively Reweighted Least Square; OR: Odds ratio; WHO: World Health Organization

\section{Introduction}

World Health Organization (WHO) defined acute respiratory infection (ARI) as "a clinical state presenting with rapid breathing more than expected upper limit for age with or without chest in drawing, too sick to feed, nasal discharge, cough, fever with or without auscultatory findings of less than 2 weeks." The ARI symptoms include a cough accompanied by short, rapid breathing that is chest-related and/or difficult breathing that is chest-related. Acute respiratory infections (ARI) are found in either the upper or lower respiratory tract. Signs and symptoms for upper respiratory tract infections include common cold, frequent sneezing, restlessness and cough while that of the lower tract includes foul breath, fast breathing, difficult breathing, cough and chest in-drawing. Globally ARIs incidence is high among infants, children, and the elderly and is more pronounced in low income countries [1].

ARIs are the major cause of mortality and morbidity among under five children, especially in developing countries. Even though child mortality has been declining globally as a result of improved child health care interventions and socio-economic development, there were about 7.6 million children who died before celebrating their fifth year of birth day in 2010 [2,3] which is attributed to respiratory tract infections. Of the 7.6 million child deaths that occurred worldwide in 2010, pneumonia, diarrhea, and malaria which is a severe form of ARI were the leading causes of death, where the largest burden of mortality in under five children was reported in developing regions; Africa is accounting for 3.6 million deaths and Southeast Asia accounts about 2.1 million deaths of infants and younger children. Concerning morbidity, recent estimates suggest $3.5 \%$ of the global burden of disease is caused by ARI. In developing countries, on an average, every child has five episodes of ARI per year accounting for 30\%-50\% of the total pediatric outpatient visits $[4,5]$.

From various previous literatures and theories, it has been shown that poor socio-economic status is associated with inadequate utilization of primary health care services [6,7]. Maternal age and educational status of mothers are other characteristics of a mother

${ }^{\star}$ Correspondence to: Berhanu Teshome Woldeamanuel, Department of Statistics, Mekelle University, P.O. Box 231, Mekelle, Tigray, Ethiopia, E-mail: berteshome19@gmail.com

Key words: chest-related symptoms, acute respiratory infections, under five, logistic regression

Received: July 25, 2019; Accepted: August 07, 2019; Published: August 12, 2019 
that highly associated health care of a child. Mothers younger than 35 years and also mothers who have completed secondary education exhibit more help seeking behavior for their sick children $[6,8]$. The distribution of risk factors of acute respiratory infection varies with place of residence, mothers' education and birth order of a child [9] a similar study in Ethiopia revealed that mothers' education level, age of children, mothers occupational status, source of drinking water, type of toilet facility and economic status of family significant factor associated with symptoms of acute respiratory infections [10].

Despite being one of the fastest-growing economies in the world, the Federal Democratic Republic of Ethiopia still suffers from structural and economic problems. The deadliest diseases in Ethiopia are often preventable. However, a lack of resources can make them difficult to prevent or treat. The Global Burden of Diseases (GBD) study 2015 states that ARI was the first deadliest disease in Ethiopian accounting for $10 \%$ of deaths each year, followed by diarrhea which accounts about $8 \%$ of deaths in Ethiopia each year [11]. Recent studies indicate that Ethiopia has achieved significant results in improving child survival, where under- 5 mortality has decreased by two-thirds [12].

There are just a few studies on the risk factors of ARI among under five children in Ethiopia. Given that, in Ethiopia, ARI among under five children is the highest problem contributing to child mortality, the current study aims to assess the prevalence and associated risk factors of ARI among children. It may benefit policy makers, individual households to prevent the health of their child, researchers, and decision makers in designing interventions to promote health care service and health care seeking behavior for children with symptoms of ARI.

\section{Methods and materials}

Ethiopian Demographic and Health Survey (EDHS) 2016 is the fourth survey of its kind conducted in Ethiopia. The 2016 EDHS is a nationwide sample survey of household and women of reproductive age that provides information on childhood mortality levels, fertility preferences, use of family planning methods, and maternal, child, and newborn health, knowledge of HIV/AIDS, and prevalence of HIV/AIDS and anemia. The sample for the 2016 EDHS is nationally representative along with a representative for each nine regions and two administrative cities [13].

The prevalence of ARI was estimated based on its symptoms as reported by mothers. In the survey, mothers were asked whether their children under age of five years had been ill with a cough accompanied by short, rapid breathing which was chest related in the two weeks preceding the survey [14]. These symptoms are compatible with ARI. Thus, the dependent variable "acute respiratory infection" is binary and coded as ' 1 ' if the child suffered from ARI and ' 0 ' otherwise. From various literatures covariates like child characteristics, maternal socioeconomic, demographic characteristics, environmental and health factors are considered as key determinants of ARI among under five children.

\section{Logistic regression model}

Logistic regression is a special case of generalized linear models in which the mean of the response variable is related to covariates through a regression equation. It is used to predict a categorical response from a set of predictor variables and expresses the relation between the systematic component and the log of odds of success.

\section{Binary logistic regression model}

The dependent variable in this study, ARI is dichotomous, that is, the dependent variable can take the value " 1 " with probability of success (if the child suffered from ARI) and the value "0" with probability of failure (if the child does not suffer from ARI). The single outcome Y (ARIs) follows a Bernoulli probability function that can be coded as, 1 (if the child suffered from ARI) with probability success $\pi(\mathrm{x})$, and 0 (if the child not suffered from ARI) with probability failure $1-\pi(\mathrm{x})$.

Let $Y_{i}$ denote a binary indicator variable (ARIs) of the response for subject $\mathrm{i}\left(\mathrm{i}^{\text {th }}\right.$ child), such that

$$
\mathrm{Y}_{\mathrm{i}}=\left\{\begin{array}{l}
1 \mathrm{f} \text { the } \mathrm{i}^{\mathrm{h}} \text { child suffered from ARI } \\
0 \text { otherwise }
\end{array}\right.
$$

Let there are $\mathrm{k}$ covariates, then the logistic regression model is given as:

$$
\begin{aligned}
& \pi(\mathrm{x})=\frac{\mathrm{e}^{\left(\beta_{0}+\beta_{1} \mathrm{x}_{1}+\beta_{2} \mathrm{x}_{2}+\cdots+\beta_{\mathrm{k}} \mathrm{x}_{\mathrm{k}}\right)}}{1+\mathrm{e}^{\left(\beta_{0}+\beta_{1} \mathrm{x}_{1}+\beta_{2} \mathrm{x}_{2}+\cdots+\beta_{\mathrm{k}} \mathrm{x}_{\mathrm{k}}\right)}} \\
& \text { where, } \beta_{0}=\text { the constant of the equation } \\
& x_{\mathrm{i}}^{\prime} S=\text { are the coefficients of the predictor variable, } \mathrm{i}=1,2, \ldots, \mathrm{k} . \\
& x_{\mathrm{i}}^{\prime} S=\text { are the predictor variables, } \mathrm{i}=1,2, \ldots, \mathrm{k} .
\end{aligned}
$$

The elements of such a model are distributed for the response variables and function that links the distribution to these covariates called link function.

The logit link is defined as $\log \left(\frac{\pi}{1-\pi}\right)$ For this study the outcome (the predicted value) in logistic regression analysis is coded 0 and 1 where 1 indicates the success (the child is suffered from ARI) and 0 shows the failure (no ARI).

The ratio of probability success to probability failure $\left(\frac{\pi}{1-\pi}\right)$ is odds of success. Therefore, the logistic model can be written as:

$$
\left(\frac{\pi}{1-\pi}\right)=\exp \left\{\beta_{0}+\beta_{1} x_{1}+\cdots+\beta_{k} x_{k}\right\} \text { which means that }
$$

$\exp \left\{\beta_{i}\right\}$ where $\mathrm{i}=1,2, . ., \mathrm{k}$ is a factory by which the odds of occurrence of success changes by unit increase in the $i^{\text {th }}$ covariate.

The parameter of binary logistic model is estimated from the likelihood function of a Bernoulli distribution. Let the likelihood function of Bernoulli distribution $\mathrm{L}$.

$$
\begin{aligned}
& L=\prod_{i=1}^{n} p\left(y_{i}\right)=\prod_{i=1}^{n} \pi^{y_{i}}(1-\pi)^{\left(1-y_{i}\right)} \\
& \text { By substituting }\left(\frac{e^{x^{\prime} \beta}}{1+e^{x^{\prime} \beta}}\right) \text { to }, \text { we get } \\
& L=\prod_{i=1}^{n}\left(\frac{e^{x^{\prime} \beta}}{1+e^{x^{\prime} \beta}}\right)^{y_{i}}\left(1-\frac{e^{x^{\prime} \beta}}{1+e^{x^{\prime} \beta}}\right)^{\left(1-y_{i}\right)}
\end{aligned}
$$

To find the value of $\beta$ that maximizes the log-likelihood function we differentiate $\log L$ with respect to each $\beta$, and set the resulting expressions equal to zero. 


$$
\log L=\left(\sum_{i=1}^{n} y_{i}\right) \log \left(\frac{e^{x^{\prime} \beta}}{1+e^{x^{\prime} \beta}}\right)+\left(n-\sum_{i=1}^{n} y_{i}\right) \log \left(1-\frac{e^{x^{\prime} \beta}}{1+e^{x^{\prime} \beta}}\right)
$$

Since the expressions are non-linear in the parameters, the solution for the maximum likelihood estimates is obtained by a method called Newton Raphson iteration which is known as Iteratively Reweighted Least Square (IRLS) algorithm [15]

\section{Tests of model goodness of fit}

The goodness of fit of a model measures how well the model describes the response variable. Assessing goodness of fit involves investigating how close values predicted by the model with that of observed values [16].

For the $\mathrm{k}$ covariates, the over all of goodness the model can be checked by testing the following:

$$
\begin{aligned}
& H_{0}=\beta_{1}=\beta_{2}=\cdots=\beta_{k}=0 \\
& H_{1}=\beta_{i} \neq 0 \text { at least for one } \mathrm{i} \text {; where } \mathrm{i}=1,2 \ldots \mathrm{k} .
\end{aligned}
$$

Several test statistics had used for the above hypothesis. Some of these are:

\section{Pearson's Chi-square}

The goodness of fit test usually tested by Pearson's chi-square test which is the sum of differences between observed and expected outcomes (that is, counts of observations), each squared and divided by the expectation;

$$
\chi^{2}=\sum_{i=1}^{n} \frac{\left(O_{i}-E_{i}\right)^{2}}{E_{i}}
$$

Where $O_{i}=$ is the observed counts and $E_{i}=$ is the corresponding expected class frequencies.

This Pearson chi-square is compared with the chi-square distribution with $k$ - 1 degrees of freedom. The null hypothesis that states all the covariates are insignificant is rejected for large value of Pearson chi-square.

\section{Likelihood-Ratio Test}

A very important used approach to test the significance of a number of covariates is to use the likelihood ratio test. The likelihood ratio test is better, particularly if the sample size is large or the parameters are small $[15,16]$. The likelihood-ratio test uses the ratio of the maximized value of the likelihood function for the full model $\left(l_{1}\right)$ over the maximized value of the likelihood function for the simpler model $\left(l_{0}\right)$. The likelihoodratio test statistic is defined as:

$$
-2 \log \left(\frac{l_{0}}{l_{1}}\right)=-2\left[\log \left(l_{0}\right)-\log \left(l_{1}\right)\right]
$$

This log transformation of the likelihood functions yields a chisquared statistic with 1 degree of freedom, and we shall reject the null hypothesis for large value of likelihood-ratio test.

\section{The Hosmer-Lemeshow Test}

The other test used to measure of the model fit is the Hosmer and Lemeshow goodness-of-fit statistic, which measures the correspondence between the actual and predicted values of the dependent variable. The Hosmer-Lemeshow test is a commonly used test for assessing the goodness of fit of a model and allows for any number of covariates, which may be continuous or categorical. The test divides the subjects into deciles based on predicted probabilities and then computes a chisquare from observed and expected frequencies.

The hypothesis to be tested is:

$\mathrm{H}_{\mathrm{o}}$ : The model fit the data.

$\mathrm{H}_{1}$ : The model does not fit the data.

The Hosmer-Lemeshow test is given by:

$H_{0}=\beta_{i}=0$

Where $O_{k}=$ the observed number of events in the $\mathrm{k}^{\text {th }}$ group.

$E_{k}=$ the expected value of the events in the $\mathrm{k}^{\text {th }}$ group.

$V_{k}=$ the variance correction factor for the $\mathrm{k}^{\text {th }}$ group.

The Hosmer-Lemeshow test is compared with chi-square distribution with 8 degrees of freedom. The small value of HosmerLemeshow $(\hat{C})$ shows that the model fits the data.

\section{Wald Test}

Wald test used to test the statistical significance of each coefficient $(\beta)$ in the model.

The hypothesis to be tested is:

$H_{0}=\beta_{i}=0$ (the $\mathrm{i}^{\text {th }}$ covariate is insignificant).

$H_{1}=\beta_{i} \neq 0$ (the ${ }^{\text {ith }}$ covariate is significant); $\mathrm{i}=1,2, \ldots, \mathrm{k}$

The test statistic is:

$$
w=\left(\frac{\hat{\beta}_{i}}{\mathcal{E}\left(\hat{\beta}_{i}\right)}\right)^{2}
$$

The Wald test $(w)$ has a chi-square distribution with 1 degree of freedom. Based on Wald statistic we reject the null hypothesis for large value of $w$.

\section{Results}

\section{Results of descriptive statistics}

Table 1 presents summary statistics of the distribution of ARIs by different socio economic, demographic and environmental factors in the study area. The total number of children covered in the present study was 6719 . The results reveal that about $10.4 \%$ of under-five children suffer from ARI during two weeks preceding the survey. Considering the ARI distribution by regions Oromia and Tigray accounts for the highest proportion of ARIs $2.5 \%$ and $2 \%$, respectively (Table 1 ).

The result shows the prevalence ARIs of differing by type of place of residence. Accordingly, the percentage of acute respiratory infected children in the rural area are 5.5 times more than, that of the urban counterparts. Concerning family demographic and socioeconomic status children ARIs status also differs by maternal education level, age of mothers, household economic level and partners/husband education. Children born to mothers with no education have the highest percentage of suffering from ARI $6.4 \%$ and that of primary education is $3.2 \%$. Generally as mothers get more educated the probability that their children are being infected by acute respiratory infections decrease. This figure also consistent as partners' education is concerned; i.e. compared to those with secondary and above education level children to mothers whose partner is illiterate or has primary education has high proportion of ARI. 
Woldeamanuel BT (2019) Prevalence and risk factors for chest-related symptoms of acute respiratory tract infections among under five children: Case of Ethiopia

Table 1. Summary statistics of socio-economic, demographic and environmental and health factors that are assumed to associate with ARIs among under five children in Ethiopia

\begin{tabular}{|c|c|c|c|c|}
\hline \multirow{2}{*}{ Covariates } & \multirow{2}{*}{ Categories } & \multicolumn{2}{|c|}{ Acute respiratory infection } & \multirow{2}{*}{ Total } \\
\hline & & No & Yes & \\
\hline \multirow{7}{*}{ Maternal Age } & $15-19$ & $294(4.4 \%)$ & $32(0.5 \%)$ & $326(4.9 \%)$ \\
\hline & $20-24$ & $1232(18.3 \%)$ & $163(2.4 \%)$ & $1395(20.8 \%)$ \\
\hline & $25-29$ & $1692(25.2 \%)$ & $191(2.8 \%)$ & $1883(28.0 \%)$ \\
\hline & $30-34$ & $1295(19.3 \%)$ & $151(2.2 \%)$ & $1446(21.5 \%)$ \\
\hline & $35-39$ & $971(14.5 \%)$ & $98(1.5 \%)$ & $1069(15.9 \%)$ \\
\hline & $40-44$ & $402(6.0 \%)$ & $47(0.7 \%)$ & $449(6.7 \%)$ \\
\hline & $45-49$ & $135(2.0 \%)$ & $16(0.2 \%)$ & $151(2.2 \%)$ \\
\hline \multirow{11}{*}{ Region } & Tigray & $592(8.8 \%)$ & $135(2 \%)$ & $727(10.8 \%)$ \\
\hline & Afar & $546(8.1 \%)$ & $59(0.9 \%)$ & $605(9 \%)$ \\
\hline & Amhara & $625(9.3 \%)$ & $88(1.3 \%)$ & $713(10.6 \%)$ \\
\hline & Oromia & $783(11.7 \%)$ & $166(2.5 \%)$ & $949(14.1 \%)$ \\
\hline & Somali & $706(10.5)$ & $44(0.7 \%)$ & $750(11.2 \%)$ \\
\hline & Benishangul & $534(7.9 \%)$ & $17(0.3 \%)$ & $551(8.2 \%)$ \\
\hline & SNNPR & $751(11.2 \%)$ & $97(1.4 \%)$ & $848(12.6 \%)$ \\
\hline & Gambela & $459(6.8 \%)$ & $36(0.5 \%)$ & $495(7.4 \%)$ \\
\hline & Harari & $373(5.6 \%)$ & $13(0.2 \%)$ & $386(5.7 \%)$ \\
\hline & Addis Ababa & $322(4.8 \%)$ & $25(0.4 \%)$ & $347(5.2 \%)$ \\
\hline & Dire Dawa & $330(4.9 \%)$ & $18(0.3 \%)$ & $348(5.2 \%)$ \\
\hline \multirow{2}{*}{ Place of residence } & Urban & $1297(19.3 \%)$ & $107(1.6 \%)$ & $1404(20.9 \%)$ \\
\hline & Rural & $4724(70.3 \%)$ & $591(8.8 \%)$ & $5315(79.1 \%)$ \\
\hline \multirow{4}{*}{ Maternal education level } & No education & $3640(54.2 \%)$ & $433(6.4 \%)$ & $4073(60.6 \%)$ \\
\hline & Primary & $1593(23.7 \%)$ & $215(3.2 \%)$ & $1808(26.9 \%)$ \\
\hline & Secondary & $511(7.6 \%)$ & $30(0.4 \%)$ & $541(8.1 \%)$ \\
\hline & Higher & $277(4.1 \%)$ & $20(0.3 \%)$ & $297(4.4 \%)$ \\
\hline \multirow{2}{*}{ Sex of household head } & Male & $4651(69.2 \%)$ & $589(8.8 \%)$ & $5240(78 \%)$ \\
\hline & Female & $1370(20.4 \%)$ & $109(1.6 \%)$ & $1479(22 \%)$ \\
\hline \multirow{2}{*}{ Sex of child } & Male & $3085(45.9 \%)$ & $360(5.4 \%)$ & $3445(51.3 \%)$ \\
\hline & Female & $2936(43.7 \%)$ & $338(5 \%)$ & $3274(48.7 \%)$ \\
\hline \multirow{2}{*}{ Breast feeding status } & No & $2320(34.5 \%)$ & $221(3.3 \%)$ & $2541(37.8 \%)$ \\
\hline & Yes & $3701(55.1 \%)$ & $477(7.1 \%)$ & $4178(62.2 \%)$ \\
\hline \multirow{2}{*}{ Had fever in last two weeks } & No & $5371(79.9 \%)$ & $264(3.9 \%)$ & $5635(83.9 \%)$ \\
\hline & Yes & $650(9.7 \%)$ & $434(6.4 \%)$ & $1084(16 \%)$ \\
\hline \multirow{2}{*}{ Had cough in last two weeks } & No & $5418(80.6 \%)$ & $78(1.2 \%)$ & $5496(81.8 \%)$ \\
\hline & Yes & $602(9 \%)$ & $620(9.2 \%)$ & $1222(18.2 \%)$ \\
\hline \multirow{3}{*}{ Source of drinking water } & Piped & $1908(28.4 \%)$ & $221(3.3 \%)$ & $2129(31.7 \%)$ \\
\hline & Tube-well & $870(12.9 \%)$ & $100(1.5 \%)$ & $970(14.4 \%)$ \\
\hline & Others & $3243(48.3 \%)$ & $377(5.6 \%)$ & $3620(53.9 \%)$ \\
\hline \multirow{2}{*}{ Toilet facility } & No facility/ bush/field & $2644(39.4 \%)$ & $305(4.5 \%)$ & $2949(43.9 \%)$ \\
\hline & Standard/with facility & $3377(50.3 \%)$ & $393(5.8 \%)$ & $3770(56.1 \%)$ \\
\hline \multirow{3}{*}{ Household size } & $1-4$ & $1919(28.6 \%)$ & $221(3.3 \%)$ & $2140(31.8 \%)$ \\
\hline & $5-7$ & $2792(41.6 \%)$ & $333(5 \%)$ & $3125(46.5 \%)$ \\
\hline & 8 and more & $1310(19.5 \%)$ & $144(2.1 \%)$ & $1454(21.6 \%)$ \\
\hline \multirow{2}{*}{ Working status of mother } & Not working & $3465(51.6 \%)$ & $367(5.5 \%)$ & $3832(57 \%)$ \\
\hline & Working & $2556(38 \%)$ & $331(4.9 \%)$ & $2887(43 \%)$ \\
\hline \multirow{4}{*}{ Husband/partners education } & No education & $2681(42.9 \%)$ & $304(4.8 \%)$ & $2985(47.8 \%)$ \\
\hline & Primary & $1741(27.9 \%)$ & $274(4.4 \%)$ & $2015(32.2 \%)$ \\
\hline & Secondary & $649(10.4 \%)$ & $59(0.9 \%)$ & $708(11.3 \%)$ \\
\hline & Higher & $518(8.3 \%)$ & $25(0.4 \%)$ & $543(8.7 \%)$ \\
\hline & 1 & $1201(17.9 \%)$ & $151(2.2 \%)$ & $1352(20.1 \%)$ \\
\hline Birth order & $2-3$ & $1884(28 \%)$ & $197(2.9 \%)$ & $2081(31 \%)$ \\
\hline Birth oraer & $4-6$ & $1887(28.1 \%)$ & $217(3.2 \%)$ & $2104(31.3 \%)$ \\
\hline & 7 and more & $1049(15.6 \%)$ & $133(2 \%)$ & $1182(17.6 \%)$ \\
\hline & Poor & $3045(45.3 \%)$ & $330(4.9 \%)$ & $3375(50.2 \%)$ \\
\hline Wealth index & Middle & $827(12.3 \%)$ & $135(2 \%)$ & $962(14.3 \%)$ \\
\hline & Rich & $2149(32 \%)$ & $233(3.5 \%)$ & $2382(35.5 \%)$ \\
\hline
\end{tabular}




\begin{tabular}{|c|c|c|c|c|}
\hline \multirow{8}{*}{ Age of child in months } & Less than 6 & $975(14.5 \%)$ & $102(1.5 \%)$ & $1077(16 \%)$ \\
\hline & $6-11$ & $869(12.9 \%)$ & $118(1.8 \%)$ & $987(14.7 \%)$ \\
\hline & $12-17$ & $930(13.8 \%)$ & $133(2 \%)$ & $1063(15.8 \%)$ \\
\hline & $18-23$ & $653(9.7 \%)$ & $77(1.1 \%)$ & $730(10.9 \%)$ \\
\hline & $24-29$ & $731(10.9 \%)$ & $84(1.3 \%)$ & $815(12.1 \%)$ \\
\hline & $30-35$ & $483(7.2 \%)$ & $53(0.8 \%)$ & $53(0.8 \%)$ \\
\hline & $36-41$ & $470(7 \%)$ & $41(0.6 \%)$ & $511(7.6 \%)$ \\
\hline & 42 and higher & $910(13.5 \%)$ & $90(1.3 \%)$ & $1000(14.9 \%)$ \\
\hline Total & & $6021(89.6 \%)$ & $698(10.4)$ & $6719(100 \%)$ \\
\hline
\end{tabular}

Another factor that shows high variation in under five children ARI status is household wealth index. Tables 1 reveal that the poor families account for the higher proportion ARI among under five children. As family size, i.e. number of household members and child birth order concerned, the highest percentage ARI among under five children is observed for family with 5-7 household members, in which $5 \%$ are suffered from ARI. For birth order number of children with birth order number 4-6 and 2-3 accounts for the highest proportion of acute respiratory infections, of those children with birth order number 4-6, $3.2 \%$, were suffered from ARI, and $2.9 \%$ are from those children with birth order number of 2-3 respectively.

With regard to child sex prevalence of ARI among male children is nearly greater than that of females. The prevalence of ARI also varies according to age of a child. Children under the age of 17 months account for the highest percentage of ARI compared that of older age children. This may be the reason that younger infants are less able to resist infection and could be easily caught by cough and diarrhea.

\section{Results of multivariable binary logistic regression model}

Logistic regression analysis was used to identify the risk factors of ARI. The model goodness of fit test was done using Hosmer and Lemeshow test and the statistical test of significance of individual coefficients of each ARI symptoms among under five children were made based on Wald Chi-square. The Hosmer and Lemeshow test evaluate the goodness of fit between predicted and observed probabilities in classifying the response variable. The Hosmer and Lemeshow test value suggests the model is a good fit to the data (chi-square $=7.470, \mathrm{p}=$ 0.487 ), i.e. the probabilities of predicted versus observed values of the response variable match up as well as we would like. Thus, our fitted logistic regression model is good fit.

A binary logistic regression result in Table 2 provides estimates of the effect of some selected risk factors of ARI among children. The result revealed that place of residence, sex of household head, education level of mother, partners education, baby postnatal checkup, source of drinking water, age of a child in months, and working status of mother are found to be the significant risk factors for occurrence of ARI symptoms among under five children at $5 \%$ level of significance.

While the variables sex of a child, availability of toilet facility, wealth index, age of mother at first birth, family size, and birth order of a child are not statistically significant at $5 \%$ level of significance.

Children living in the rural areas had an increased risk to ARI symptoms compared to those children living in the urban areas. The findings of the study in Table 2 show that the odds of suffering from ARIs among under five children born to a mother living the urban area was associated with a $33.2 \%$ decreased risk of having ARIs symptoms compared to being born to mothers residing in the rural area counter parts, keeping all other covariates constant. Results in Table 2 also indicate that children born from mothers whose household head is male have a significantly higher risk of ARI compared to those born from mothers whose household head is female. The risk of suffering from ARI was about $34.7 \%$ higher for births to mothers whose household head is male compared with those family whose household head is female (OR: 0.1.347; 95\% (CI: 1.061-1.711)).

Children born to a mother with no education were associated with a $52.9 \%$ increased risk of ARI symptoms compared to being born to mothers with secondary and higher education, while primary education of the mothers increases the risk of suffering from ARI symptoms by $55.9 \%$, keeping all other covariates constant. Further, Table 2 shows that children of mothers whose husband has no schooling and primary education have a significantly higher risk of ARI than children of mothers whose husband has higher education. The odds of suffering from ARIs among children with a mother whose husband had no education were associated with a $87.4 \%$ increased risk (OR: 1.874 . 95\% (CI: 1.153-3.045)) and children born to a mother whose partner has primary education are 2.378 times more likely to have symptoms of ARIs compared to being born to mothers whose partner has higher education, keeping all other covariates constant.

Children born from mothers not attending postnatal care service after delivery within two months have about $50 \%$ higher risk of ARI symptoms than those children born from mothers attending postnatal care services. Concerning sources of drinking water the odds of suffering from ARI symptoms were 1.248 times more likely to children with piped water compared to those using other sources (OR 1.248; 95\% CI (1.006-1.547)).

The study shows that current age of children is another covariate that highly related with symptoms of ARI. Compared to children whose age is 42 and above months, children whose age in the age group 6-11 months and 12-17 months are associated with increased risk of ARI symptoms (OR: 1.460; 95\% CI (1.070-1.992)), (OR: 1.552; 95\% CI (1.147-2.100)) respectively. Lastly, occupation of mothers was found to be a significant covariate of ARI symptoms among under five children. Compared to children of mothers who are currently working those children whose mother's is not working have a significantly lower risk of ARI symptoms (OR: 0.775 654; 95\% CI (0.654-0. 918)) (Table 2).

\section{Discussion}

The study has empirically investigated and identified the risk factors of acute respiratory infection symptoms, among under five children in Ethiopia based on the Ethiopian Demographic and Health Survey data $[13,14]$. Accordingly, different descriptive statistics were computed and multivariable logistic regression model was fitted to the data to identify socio-demographic, economic and environmental and health related factors of ARI symptoms among under five children in Ethiopia.

The study uses retrospective study design. The main limitation of this study is gathering all necessary variables and some variables did not get in a way we need. This may affect the association of the 
Table 2. Result of Multivariable Binary Logistic Regression Analysis of risk factors for Symptoms of ARI

\begin{tabular}{|c|c|c|c|c|c|c|c|c|}
\hline \multirow{2}{*}{ Covariates } & \multirow{2}{*}{ B } & \multirow{2}{*}{ S.E. } & \multirow{2}{*}{ Wald } & \multirow{2}{*}{ Df } & \multirow{2}{*}{ Sig. } & \multirow{2}{*}{$\operatorname{Exp}(B)$} & \multicolumn{2}{|c|}{ 95\% C.I.for EXP(B) } \\
\hline & & & & & & & Lower & Upper \\
\hline Residence (rural) & -0.403 & 0.157 & 6.613 & 1 & $.010^{*}$ & 0.668 & 0.492 & 0.909 \\
\hline Sex of household head (female) & 0.298 & 0.122 & 5.964 & 1 & $.015^{*}$ & 1.347 & 1.061 & 1.711 \\
\hline Sex of child (female) & 0.01 & 0.083 & 0.015 & 1 & 0.904 & 1.01 & 0.858 & 1.189 \\
\hline Source of drinking water (others) & & & 6.932 & 2 & $.042 *$ & & & \\
\hline Piped & 0.221 & 0.11 & 4.074 & 1 & $.044 *$ & 1.248 & 1.006 & 1.547 \\
\hline Tube-well & -0.107 & 0.126 & 0.716 & 1 & 0.397 & 0.899 & 0.702 & 1.151 \\
\hline Toilet facility (yes) & 0.139 & 0.102 & 1.853 & 1 & 0.173 & 1.149 & 0.941 & 1.402 \\
\hline Household size (8+) & & & 2.794 & 2 & 0.247 & & & \\
\hline $1-4$ & 0.182 & 0.157 & 1.336 & 1 & 0.248 & 1.199 & 0.881 & 1.631 \\
\hline $5-7$ & 0.208 & 0.124 & 2.789 & 1 & 0.095 & 1.231 & 0.965 & 1.571 \\
\hline Age at first birth $(24+)$ & & & 0.016 & 2 & 0.992 & & & \\
\hline Less than 18 years & 0.001 & 0.091 & 0 & 1 & 0.995 & 1.001 & 0.838 & 1.195 \\
\hline $18-23$ & -0.018 & 0.156 & 0.013 & 1 & 0.91 & 0.982 & 0.723 & 1.334 \\
\hline Work status of mother (working) & -0.255 & 0.087 & 8.666 & 1 & $.003 *$ & 0.775 & 0.654 & 0.918 \\
\hline Birth order $(7+)$ & & & 7.222 & 3 & 0.065 & & & \\
\hline 1 & 0.07 & 0.183 & 0.147 & 1 & 0.702 & 1.073 & 0.749 & 1.536 \\
\hline $2-3$ & -0.236 & 0.155 & 2.314 & 1 & 0.128 & 0.79 & 0.582 & 1.07 \\
\hline $4-6$ & -0.164 & 0.133 & 1.512 & 1 & 0.219 & 0.849 & 0.654 & 1.102 \\
\hline Wealth index (rich) & & & 5.676 & 2 & 0.059 & & & \\
\hline Poor & -0.212 & 0.127 & 2.808 & 1 & 0.094 & 0.809 & 0.631 & 1.037 \\
\hline Middle & 0.066 & 0.136 & 0.238 & 1 & 0.626 & 1.068 & 0.819 & 1.393 \\
\hline Child age in months $(42+)$ & & & 14.369 & 7 & $.045^{*}$ & & & \\
\hline Less than 6 & 0.142 & 0.162 & 0.768 & 1 & 0.381 & 1.153 & 0.839 & 1.585 \\
\hline $6-11$ & 0.378 & 0.159 & 5.694 & 1 & $.017^{*}$ & 1.46 & 1.07 & 1.992 \\
\hline $12-17$ & 0.439 & 0.154 & 8.098 & 1 & $.004 *$ & 1.552 & 1.147 & 2.1 \\
\hline $18-23$ & 0.237 & 0.173 & 1.879 & 1 & 0.171 & 1.268 & 0.903 & 1.781 \\
\hline $24-29$ & 0.178 & 0.171 & 1.076 & 1 & 0.3 & 1.194 & 0.854 & 1.67 \\
\hline $30-35$ & 0.153 & 0.192 & 0.635 & 1 & 0.426 & 1.165 & 0.8 & 1.696 \\
\hline $36-41$ & -0.053 & 0.206 & 0.065 & 1 & 0.798 & 0.949 & 0.634 & 1.42 \\
\hline Maternal education (secondary \& higher) & & & 5.034 & 2 & 0.081 & & & \\
\hline No education & 0.425 & 0.211 & 4.066 & 1 & $.044 *$ & 1.529 & 1.012 & 2.31 \\
\hline Primary education & 0.444 & 0.199 & 5.003 & 1 & $.025 *$ & 1.559 & 1.056 & 2.301 \\
\hline Post-natal care (yes) & 0.442 & 0.132 & 11.255 & 1 & $.001 *$ & 1.643 & 1.497 & 1.832 \\
\hline Husband education level (higher) & & & 18.194 & 3 & $.000^{*}$ & & & \\
\hline No education & 0.628 & 0.248 & 6.43 & 1 & $.011^{*}$ & 1.874 & 1.153 & 3.045 \\
\hline Primary education & 0.866 & 0.241 & 12.945 & 1 & $.000^{*}$ & 2.378 & 1.484 & 3.813 \\
\hline Secondary education & 0.489 & 0.254 & 3.685 & 1 & 0.055 & 1.63 & 0.99 & 2.684 \\
\hline Constant & -3.11 & 0.343 & 82.04 & 1 & $.000 *$ & 0.045 & & \\
\hline
\end{tabular}

Reference categories are in parentheses

* Significant at $5 \%$ level

outcome variable and the determinant factors. The confounding effect of unmeasured variables cannot be controlled. Some determinant factors like maternal vaccination and diarrhea status are important determinant factors, but we fail to include them in the analysis due to incompleteness of the data. Thus, any reader of this article should take in to account the above limitations.

The odds of ARI symptoms were less likely among children born to mothers living in the urban area, such that children living in the rural areas had an increased risk to suffer from acute respiratory infection symptoms compared to those children living in the urban areas. Theoretically, all things being equal, living in urban areas should be associated with a higher standard of living, better sanitation, and better health facilities, among other things [17]. Descriptive statistics results also have revealed that ARI symptom is higher in rural areas of the study area.

For the variable sex of household head the odds of acute respiratory infections is less to mothers whose household head is female than the comparison category whose household head is male. As maternal education is concerned the findings of the study show that there is a significant difference in the risk of acute respiratory infections by mothers' educational level. The risk of ARI symptoms is, significantly higher for children whose mothers have no education and primary education level than children whose mothers have secondary and higher level of education. Moreover educational status of mothers appears to be related to health care seeking behavior $[8,18]$. This may be an indication that education improves the ability of mothers to implement simple health knowledge and facilitates their capacity to manipulate their environment including health care facilities, interact more effectively with health professionals, comply with treatment recommendations, and keep their environment clean. Furthermore, educated women have greater control over health choices for their children. Similarly regarding education level of a husband/partner the result reveals that children from families with an educated father has a lower chance of being suffering from acute respiratory infections [19]. 
Another important demographic factor which significantly associated with ARI is source of drinking water. The findings of this study show that children who use water from piped source sources are, highly more likely to have symptoms of acute respiratory infections than those who use other sources as drinking water. This finding is inconsistent with previous study that states access to unsafe water is the main cause of infectious diseases such as diarrhea and intestinal parasites.

Age of a child in months is another significantly correlated factor of acute respiratory infections. At the earlier age of the child the odds of symptoms of acute respiratory infection is high compared to those more than 42 months. Theoretically, as it is clear that as the age child gets older they will gain more to resistance to infections, like cough and diarrhea $[17,18,20]$. Finally, occupation of mothers was found to be a significant predictor of risk factors of acute respiratory infections. Compared to children of mothers currently working those children whose mother's is not working on any sectors have a significantly lower risk of acute respiratory infections symptom. This is if the mother lives at home she may keep her child with a good and clear environment [18].

\section{Conclusions}

The study has empirically investigated and identified the risk factors that are associated with the risk of acute respiratory infection in Ethiopia based on Ethiopian Demographic and Health Survey 2016 data using multivariable binary logistic regression analysis. According to the results of multivariable logistic regression model, it is found that the variables place of residence, sex of household head, educational level the mother attained, husband/partner's educational attainment, postnatal care after birth, source of drinking water supply, working status of mother and current age of a child in months are the significant risk factors associated with symptoms of acute respiratory infections in Ethiopia. However, sex of a child, access to toilet facility, family size, age of mother at first birth, economic status of household and birth order number of a child were found to be insignificant factors of acute respiratory infections symptom in Ethiopia.

\section{Availability of data and materials}

The data sets used to support the findings of this study are available from the corresponding author upon reasonable request.

\section{Conflicts of interest}

The authors declare no potential conflicts of interest with respect to the research, authorship, and/or publication of this article.

\section{Authors' contributions}

Berhanu Teshome Woldeamanuel conceptualized the proposal, identified and reviewed all papers, and prepared the manuscript. Haftu Legesse Gebreyesus reviewed drafts of the paper and edited the manuscript. All authors reviewed the manuscript critically for content and approved the final version to be submitted.

\section{Acknowledgements}

We wish to acknowledge Macro International, USA for allowing us access to the 2016 Ethiopian Demographic and Health Survey dataset.

\section{References}

1. Nair GB, Niederman MS (2011) Community-acquired pneumonia: an unfinished battle. Med Clin North Am 95: 1143-1161.

2. Black RE, Cousens S, Johnson HL, Lawn JE, Rudan I, et al. (2010) Global, regional, and national causes of child mortality in 2008: a systematic analysis. Lancet 375 : 1969-1987.

3. Liu L, Johnson HL, Cousens S, Perin J, Scott S, et al. (2012) Global, regional, and national causes of child mortality: an updated systematic analysis for 2010 with time trends since 2000. Lancet 379: 2151-2161.

4. Rudan I, Boschi-Pinto C, Biloglav Z, Mulholland K, Campbell H (2008) Epidemiology and etiology of childhood pneumonia. Bull World Health Organ 86: 408-416.

5. Kuruvilla S, Schweitzer J, Bishai D, Chowdhury S, Caramani D, et al. (2014) Success factors for reducing maternal and child mortality. Bull World Health Organ 92: 533544

6. Taffa N, Chepngeno G (2005) Determinants of health care seeking for childhood illnesses in Nairobi slums. Trop Med Int Health 10: 240-245.

7. Tessema F, Asefa M, Ayele F (2002) Mothers' Health Services Utilization and Health Care Seeking Behavior During Infant Rearing: A Longitudinal Community Based Study, South West. Ethiop J Heal Dev 2002: 51-58.

8. Manna B, Nasrin D, Kanungo S, Roy S, Ramamurthy T, et al. (2013) Determinants of health care seeking for diarrheal illness in young children in urban slums of Kolkata, India. Am J Trop Med Hyg 89: 56-61.

9. Kumar SG, Majumdar A, Kumar V, Naik BN, Selvaraj K, et al. (2015) Prevalence of acute respiratory infection among under-five children in urban and rural areas of puducherry, India. J Nat Sci Biol Med 6: 3-6.

10. Jabessa S (2015) Multilevel Analysis of Acute Respiratory Infection Symptoms among under Five Children in Ethiopia. J Biom Biostat 6: 251.

11. GBD 2015 Child Mortality Collaborators (2016) Global, regional, national, and selected subnational levels of stillbirths, neonatal, infant, and under-5 mortality, 1980 2015: a systematic analysis for the Global Burden of Disease Study 2015. Lancet 388 : 1725-1774.

12. Hailemariam D (2016) Sustaining gains in child health and HIV-related MDGs in Ethiopia: lessons from field research. Ethiopian J Health Dev (EJHD) 23: 2.

13. Central Statistical Agency [Ethiopia] and ICF International (2017) Ethiopia Demographic and Health Survey 2016. Addis Ababa, Ethiopia and Calverton, Maryland, USA.

14. Central Statistical Agency (CSA) [Ethiopia] and ICF (2016). Ethiopia Demographic and Health Survey 2016. Addis Ababa, Ethiopia, and Rockville, Maryland, USA: CSA and ICF.

15. AGRESTI an introduction to categorical data analysis: Florida, 1990.

16. Hosmer D, Lemeshow S (2000) Applied Logistic Regression (2nd Edn) John Wiley and sons, Inc. New York.

17. Harerimana JM, Nyirazinyoye L, Thomson DR, Ntaganira J (2016) Social, economic and environmental risk factors for acute lower respiratory infections among children under five years of age in Rwanda. Arch Public Health 74: 19.

18. Ángela María Pinzón-Rondón, Paula Aguilera-Otalvaro, Carol Zárate-Ardila, Alfonso Hoyos-Martínez (2016). Acute respiratory infection in children from developing nations: a multi-level study. Paediatr Int Child Health 36: 84-90,

19. Geberetsadik A, Worku A, Berhane Y (2015). Factors associated with acute respiratory infection in children under the age of 5 years: evidence from the 2011 Ethiopia Demographic and Health Survey. Pediatric Health Med Ther 6: 9-13.

20. Koch A, Mølbak K, Homøe P, Sørensen P, Hjuler T, et al. (2003). Risk Factors for acute respiratory tract infections in young Greenlandic children. Am J Epidemiol 158: 374-384

Copyright: (C2019 Woldeamanuel BT. This is an open-access article distributed under the terms of the Creative Commons Attribution License, which permits unrestricted use, distribution, and reproduction in any medium, provided the original author and source are credited. 